\title{
Breastfeeding and non-nutritive sucking patterns related to the prevalence of anterior open bite in primary dentition
}

\author{
Camila Campos ROMERO' ${ }^{1}$, Helio SCAVONE-JUNIOR², Daniela Gamba GARIB ${ }^{3}$, Flávio Augusto COTRIM-FERREIRA², \\ Rívea Inês FERREIRA²
}

\begin{abstract}
1 - DDS, MSc, Research Fellow, Department of Pediatric Dentistry and Orthodontics, University of São Paulo City (UNICID), São Paulo, SP, Brazil.
2 - DDS, MSc, PhD, Associate Professors, Department of Pediatric Dentistry and Orthodontics, University of São Paulo City (UNICID), São Paulo, SP, Brazil.

3 - DDS, MSc, PhD, Assistant Professor of Orthodontics, Hospital for Rehabilitation of Craniofacial Anomalies and Bauru School of Dentistry, University of São Paulo, Bauru, SP, Brazil.
\end{abstract}

Corresponding address: Profa. Dra. Rívea Inês Ferreira - Universidade Cidade de São Paulo - UNICID - Pós-Graduação - Programa de Mestrado em Ortodontia Rua Cesário Galeno, 448 - Bloco A. Tatuapé - São Paulo-SP - 03071-000 - Phone/Fax: + 5519 3251-2334 - e-mail: riveaines@yahoo.com

Received: June 24, 2009 - Modification: September 16, 2009 - Accepted: February 16, 2010

\section{ABSTRACT}

\begin{abstract}
$\mathrm{O}$ bjective: Nutritional, immunological and psychological benefits of exclusive breastfeeding for the first 6 months of life are unequivocally recognized. However, mothers should also be aware of the importance of breastfeeding for promoting adequate oral development. This study evaluated the association between breastfeeding and non-nutritive sucking patterns and the prevalence of anterior open bite in primary dentition. Material and Methods: Infant feeding and non-nutritive sucking were investigated in a 3-6 year-old sample of 1,377 children, from São Paulo city, Brazil. Children were grouped according to breastfeeding duration: G1 - non-breastfed, G2 - shorter than 6 months, G3 - interruption between 6 and 12 months, and G4 - longer than 12 months. Three calibrated dentists performed clinical examinations and classified overbite into 3 categories: normal, anterior open bite and deep bite. Chi-square tests $(p<0.05)$ with odds ratio $(O R)$ calculation were used for intergroup comparisons. The impact of breastfeeding and non-nutritive sucking on the prevalence of anterior open bite was analyzed using binary logistic regression. Results: The prevalence estimates of anterior open bite were: $31.9 \%(\mathrm{G} 1), 26.1 \%(\mathrm{G} 2), 22.1 \%$ (G3), and $6.2 \%(G 4)$. G1 would have significantly more chances of having anterior open bite compared with $\mathrm{G} 4$; in the total sample $(\mathrm{OR}=7.1)$ and in the subgroup without history of non-nutritive sucking ( $O R=9.3)$. Prolonging breastfeeding for 12 months was associated with a 3.7 times lower chance of having anterior open bite. In each year of persistence with non-nutritive sucking habits, the chance of developing this malocclusion increased in 2.38 times. Conclusions: Breastfeeding and non-nutritive sucking durations demonstrated opposite effects on the prediction of anterior open bite. Non-breastfed children presented significantly greater chances of having anterior open bite compared with those who were breastfed for periods longer than 12 months, demonstrating the beneficial influence of breastfeeding on dental occlusion.
\end{abstract}

Key words: Breastfeeding. Malocclusion. Primary dentition.

\section{INTRODUCTION}

In addition to the well-recognized nutritional, immunological and psychological benefits, breastfeeding promotes adequate development of the oral myofunctional structures $3,6,8,10,11,13,14,19,21,24$. Moreover, the association between short breastfeeding duration and increased prevalence of non-nutritive sucking habits has already been demonstrated $1,7,12,14,16,20$. Several authors have also reported the close relationship between persistent non-nutritive sucking habits and the development of malocclusions s, $2,8,12,15-17,21,22,25$.

Although shorter breastfeeding practices play an indirect role in the etiology of malocclusions, the extent to which breastfeeding duration 
periods and the development of malocclusions in primary dentition are associated still remains a matter of concern. Warren and Bishara ${ }^{22}$ (2002) found no statistically significant differences in the comparisons of mean dental arch measurements and occlusal traits of non-breastfed children and those who were breastfed over three periods: shorter than 6 months, from 6 to 12 months, and longer than 12 months. However, in two Brazilian studies ${ }^{16,17}$, posterior crossbite and anterior open bite were associated with breastfeeding for less than 9 months.

Controversial findings may be related to a positive history of persistent non-nutritive sucking habits while breastfeeding. The present study evaluated the association between different breastfeeding patterns and prevalence of anterior open bite, in primary dentition, considering not only a sample population in which persistent nonnutritive sucking appears to be a cultural feature, but also a subgroup of children without history of pacifier use and/or finger-sucking habits.

\section{MATERIAL AND METHODS}

This cross-sectional study is in agreement with Resolution 196/96 of the National Health Council/ Health Department (Brazil).

Mothers of 2,700 children aged 3 to 6 years, of both genders, attending 11 public preschools in the eastern region of São Paulo city, Southeastern Brazil, received an informed consent form and a questionnaire. All mothers were asked to sign the form and then complete the questionnaire, consisting of socio-demographic items, involving their children's name, date of birth, gender, as well as questions regarding both method and duration of infant feeding. Concerning the non-nutritive sucking behaviors, mothers were asked whether their children regularly sucked on pacifiers and/or developed finger-sucking habits.

Before this study began, the consistency of questionnaire interpretation was investigated in a group of 30 mothers, who were asked to answer all the proposed questions twice. An interval of at least two months was allowed to elapse between the first and second inquiries. The reliability of the mothers' answers was estimated by calculating the intraoperator Kappa concordance index ( $\kappa)$. All studied variables presented a high degree of reliability, with $\kappa$ values of approximately 0.957 . These questionnaires were not included in the present study sample, because the group of 30 mothers only participated in a pilot test.

From the preliminary study sample, 1,323 mother-child dyads (49\%) were excluded because: 945 mothers did not return the questionnaires, 22 mothers did not sign the free and informed consent form, 71 questionnaires were incomplete or answered in an ambiguous fashion that precluded analyses, and 285 children did not meet the inclusion criteria or refused the clinical examination. Hence, the final study sample comprised 1,377 children. A minimum sample calculation was made, with a confidence level pre-set at 95\%. For a $1 \%$ margin of error, a minimum corresponding sample size would be equal to 722 . In order to raise the exactness of the estimates, the sample was enlarged.

All children included in the sample met the following criteria: good acceptance during the clinical examinations; complete primary dentition, without permanent teeth erupted or in eruption; absence of extensive caries lesions, crown destruction or proximal restorations that could compromise occlusion; absence of early loss of primary teeth and/or dental anomalies, and absence of orofacial clefts or any other developmental anomalies that could be detrimental to breastfeeding and dental occlusion. The children had never undergone orthodontic or speech therapy. Demographic and socioeconomic statistical data for the areas where the preschools were located had shown that the study population was on a low-to-middle income level and consisted of a culturally homogeneous sample, as they were all under the same ethnic and sociocultural influences.

In the study sample, the percentages of boys and girls were very close $(50.1 \%$ and $49.9 \%$, respectively). Nevertheless, the frequencies of boys and girls varied according to the age group. The presence of boys was predominant in the age groups of $3(58.4 \%)$ and $6(55.6 \%)$ years. On the other hand, girls represented slightly higher percentages than boys in the age groups of 4 $(51.5 \%)$ and $5(52.8 \%)$ years.

Three calibrated dentists, who were blind to the questionnaire data, performed the clinical examinations to assess overbite. Training and calibration involved occlusal examinations of 24 children, who were not included in the sample, twice, with an interval of 15 days between the assessments. The degree of intraexaminer agreement was analyzed by means of Kappa statistics. Spearman's correlation tests were used for assessing the consistency of diagnoses performed by pairs of examiners. Kappa ( $\kappa)$ coefficients ranging from 0.89 to 1.00 were obtained for the three dentists, indicating very good agreement between data observed in the first and second clinical examinations. Furthermore, for the inter-examiner evaluation, correlation coefficients (Rs) higher than 0.90 were obtained for each pair of examiners, indicating the homogeneity in the diagnoses recorded.

Clinical examinations were performed in the school setting, with the child comfortably seated 
under a suitable source of artificial light. During the clinical examinations, the children were asked to sit in an upright position and bite in maximal intercuspation. By visual inspection, overbite characteristics were classified into 3 categories $^{4}$ : normal, negative (anterior open bite) and increased (deep bite). In this study, normal overbite was diagnosed whenever the incisal edges of the mandibular primary incisors contacted the palatal surfaces of the maxillary primary incisors, and about one third of the mandibular incisor crowns were covered; or whenever the incisal edges of the mandibular primary incisors contacted the incisal edges of the maxillary primary incisors. Negative overbite or anterior open bite was recorded as a lack of vertical contact between the maxillary and mandibular primary incisors. On the other hand, increased overbite or deep bite was defined as excessive vertical overlap.

After clinical examinations and based on the inclusion criteria, children were assigned to 4 groups

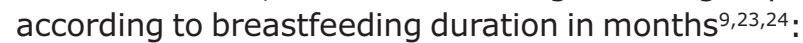
G1 - non-breastfed (comprised children who were never breastfed or those children who were breastfed for a period shorter than 1 month); G2 - breastfeeding for a period shorter than 6 months of age; G3 - breastfeeding discontinued between 6 and 12 months of age; and G4 - breastfeeding for a period longer than 12 months of age. According to the World Health Organization (WHO) ${ }^{24}$ (2006), exclusive breastfeeding is related to nutritive suckling on the mother's breast, whenever the child receives only breast milk and no other liquids or solids. Predominant breastfeeding, however, is related to nutritive suckling on the mother's breast with the occasional administration of liquids/ supplements by means of infant feeding methods that preclude artificial nipples, such as cupfeeding. Breastfeeding with occasional complementation may be done by offering liquids from a spoon or mug, but breast milk is considered the infant's predominant source of nourishment ${ }^{24}$. There also might be cases in which semisolid or solid foods are gradually introduced as the child grows and the teeth erupt, but breastfeeding still continues. In this situation, the infant feeding method may be designated as complementary breastfeeding ${ }^{14}$.
None of these categories were used in an isolated manner, as the study design comprised a variety of time intervals relative to breastfeeding duration. In the present study, breastfeeding duration patterns comprised exclusive, predominant and complementary breastfeeding phases, irrespective of the differences between categories, since the most relevant factor was the complete avoidance of bottle-feeding.

For statistical purposes, the selected children were also assigned to 2 groups according to a registered positive history of non-nutritive sucking: No habits - without history of pacifier and/or finger-sucking habits, and With habits - comprising children with history of non-nutritive sucking habits that persisted for varying periods, from 1 to 6 years of age. If the child presented a history of the two non-nutritive sucking habits (pacifier and fingersucking), the one that lasted for the longer period was chosen for statistical analyses.

Considering that anterior open bite would be elected as an occlusal "problem" because it demands interceptive orthodontic treatment during the primary dentition phase, the frequencies of this malocclusion were calculated for the study groups.

Two-dimensional cross-tabulation was performed on questionnaire information versus clinical data. Combined data were submitted to the chi-square $\left(\chi^{2}\right)$ test in order to analyze possible associations between breastfeeding duration and prevalence of anterior open bite. $P$ values of less than 0.05 were considered statistically significant. Only for the comparisons in which the difference between groups was statistically significant, the odds ratio (OR) was calculated with $95 \%$ confidence intervals. The impact of each additional month of breastfeeding duration on the prevalence of anterior open bite, as well as the influence of each additional year of non-nutritive sucking duration, was analyzed using binary logistic regression.

\section{RESULTS}

A small group of children was not breastfed $(8.6 \%)$. On the other hand, $52.3 \%$ were exclusively or predominantly breastfed for periods shorter than 6 months. Sample distribution according to gender

Table 1- Prevalence of anterior open bite according to breastfeeding duration in the total sample $(n=1,377)$

\begin{tabular}{lcc}
\hline Breastfeeding groups & Number (\%) of children & Number (\%) of anterior open bite diagnosis \\
\hline G1 (non-breastfed) & $119(100 \%)$ & $38(31.9 \%)$ \\
G2 (shorter than 6 months) & $720(100 \%)$ & $188(26.1 \%)$ \\
G3 (interruption between 6 and & $312(100 \%)$ & $69(22.1 \%)$ \\
12 months) & & $14(6.2 \%)$ \\
G4 (longer than 12 months) & $226(100 \%)$ & $309(22.4 \%)$ \\
Sum of groups & $1,377(100 \%)$ & \\
\hline
\end{tabular}


indicated similar behavior by the mothers of boys and girls, as far as the breastfeeding duration was concerned. The prevalence of anterior open bite in the total sample was of $22.4 \%$ (Table 1 ). Anterior open bite was 5 times more prevalent among non-breastfed children (G1) in comparison with those who were breastfed for a period longer than 12 months (G4). The association between breastfeeding duration and prevalence of anterior open bite was, to some extent, inversely proportional, since the percentage values for $\mathrm{G} 2$ and G3 were gradually lower in relation to G1. Nevertheless, the prevalence of anterior open bite calculated for G4 was much lower in comparison with the other groups (G1, G2 and G3).

According to comparisons shown in Table 2, OR values increased in an inverse relationship to the breastfeeding duration. When comparing $\mathrm{G} 1$ and G4, the highest OR was obtained. Non-breastfed children would have 7.1 more chances of presenting

Table 2-Association between breastfeeding duration and prevalence of anterior open bite, with odds ratio (OR) calculation, in the total sample $(n=1,377)$

\begin{tabular}{lcccc}
\hline Comparisons & $\chi^{2}$ & p value & OR & p value \\
\hline G1/G2 & 1.76 & 0.185 & - & - \\
G1/G3 & 4.44 & 0.035 & 1.65 & $0.035^{*}$ \\
G1/G4 & 40.22 & 0.000 & 7.10 & $0.000^{* *}$ \\
G2/G3 & 1.86 & 0.173 & - & - \\
G2/G4 & 40.59 & 0.000 & 5.35 & $0.000^{* *}$ \\
G3/G4 & 25.41 & 0.000 & 4.30 & $0.000^{* *}$ \\
\hline
\end{tabular}

G1: non-breastfed. G2: shorter than 6 months. G3: interruption between 6 and 12 months. G4: longer than 12 months. $\chi 2$ : chi-square value. ${ }^{*}: p$ value $<0.05 .{ }^{* *}: p$ value $<0.01$ anterior open bite than those who were breastfed for a period longer than 12 months. In this way, children who were breastfed for less than 6 months would present 5.35 more chances of having anterior open bite in comparison with those breastfed for periods longer than 12 months. Data from the binary logistic regression analysis ratifies the beneficial influence of prolonged breastfeeding duration on the prevalence of anterior open bite (Table 3). Applying the equation derived from the logistic regression model [OR=exponential (coefficient $X$ time in months $) ; 2.718(0.11 \times 12)=3.7]$, increasing breastfeeding duration for 12 months may be associated with a 3.7 times lower chance of having anterior open bite. This inverse relationship between breastfeeding duration and the occurrence of anterior open bite corroborates the data shown in Table 2 and can be visualized in Figure 1. It should be explained, however, that these analyses were based on the total sample, without taking into account the history of non-nutritive sucking habits.

Based on data from Table 4, 36.5\% of the children did not have any history of non-nutritive sucking habits. The highest percentage of positive history of non-nutritive sucking, as would be expected, was observed in G1 (81.5\%). Longer breastfeeding duration was significantly associated with lower chances of having non-nutritive sucking habits (Table 5). In fact, the binary logistic regression analysis demonstrated the significant influence of persistent non-nutritive sucking habits on the prevalence of anterior open bite (Table 6). Each additional year of persisting with non-nutritive sucking may be associated with a 2.38 times greater chance of having anterior open bite. The probabilities of having anterior open bite as the non-nutritive sucking habits persist are plotted in Figure 2, depicting this direct relationship.

Table 3- Binary logistic regression for analyzing the influence of breastfeeding duration on the prevalence of anterior open bite in the total sample $(n=1,377)$

\begin{tabular}{lccccc}
\hline Predictor & Coefficient & Standard Error & p value & OR & 95\% Confidence Interval \\
\hline Constant & 0.75 & 0.10 & $<0.001$ & & \\
Time in months & 0.11 & 0.02 & $<0.001$ & 1.11 & {$[1.07 ; 1.15]$} \\
\hline
\end{tabular}

Table 4- Distribution of children with positive history of non-nutritive sucking habits according to breastfeeding duration

\begin{tabular}{lcc}
\hline Breastfeeding groups & $\begin{array}{c}\text { Number (\%) of } \\
\text { children }\end{array}$ & $\begin{array}{c}\text { Number (\%) of positive history of non-nutritive } \\
\text { sucking }\end{array}$ \\
\hline G1 (non-breastfed) & $119(100 \%)$ & $97(81.5 \%)$ \\
G2 (shorter than 6 months) & $720(100 \%)$ & $559(77.6 \%)$ \\
G3 (interruption between 6 and 12 months) & $312(100 \%)$ & $180(57.7 \%)$ \\
G4 (longer than 12 months) & $226(100 \%)$ & $38(16.8 \%)$ \\
Sum of groups & $1,377(100 \%)$ & $874(63.5 \%)$ \\
\hline
\end{tabular}




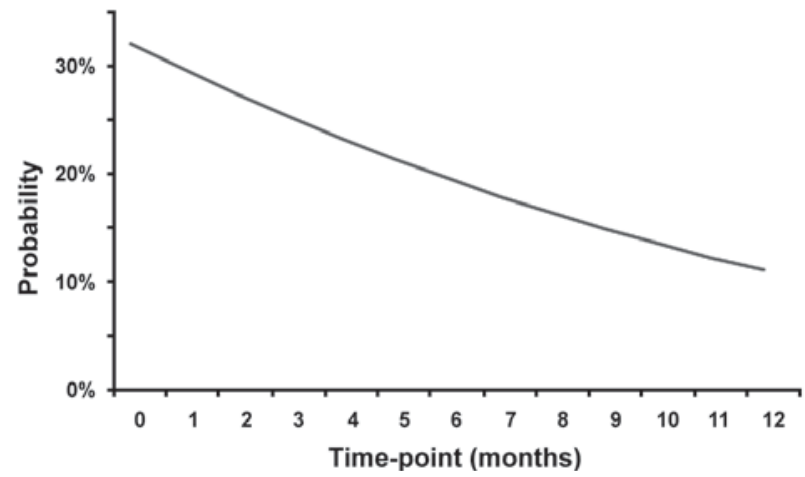

Figure 1- Relationship between breastfeeding duration and the probability of having anterior open bite (derived from the logistic regression model shown in Table 3)

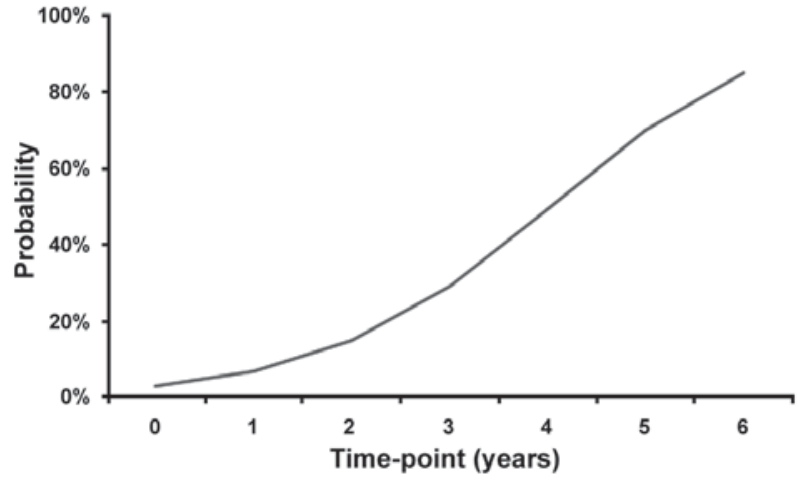

Figure 2- Relationship between non-nutritive sucking duration and the probability of having anterior open bite (derived from the logistic regression model shown in Table 6)

Table 5- Binary logistic regression for analyzing the influence of breastfeeding duration on the prevalence of non-nutritive sucking habits in the total sample $(n=1,377)$

\begin{tabular}{lccccc}
\hline Predictor & Coefficient & Standard Error & p value & OR & 95\% Confidence Interval \\
\hline Constant & -1.87 & 0.11 & $<0.001$ & & \\
Time in months & 0.24 & 0.02 & $<0.001$ & 1.27 & {$[1.24 ; 1.31]$} \\
\hline
\end{tabular}

Table 6- Binary logistic regression for analyzing the influence of non-nutritive sucking duration on the prevalence of anterior open bite in the total sample $(n=1,377)$

\begin{tabular}{lccccc}
\hline Predictor & Coefficient & Standard Error & p value & OR & 95\% Confidence Interval \\
\hline Constant & 3.52 & 0.18 & $<0.001$ & & \\
Time in years & -0.87 & 0.05 & $<0.001$ & 2.38 & {$[2.13 ; 2.63]$} \\
\hline
\end{tabular}

Table 7- Prevalence of anterior open bite according to breastfeeding duration in children without history of pacifier and/or finger-sucking habits $(n=503)$

\begin{tabular}{lcc}
\hline Breastfeeding groups & Number (\%) of children & Number (\%) of anterior open bite diagnosis \\
\hline G1 (non-breastfed) & $22(100 \%)$ & $2(9.09 \%)$ \\
G2 (shorter than 6 months) & $161(100 \%)$ & $3(1.86 \%)$ \\
G3 (interruption between 6 and 12 months) & $132(100 \%)$ & $3(2.27 \%)$ \\
G4 (longer than 12 months) & $188(100 \%)$ & $2(1.06 \%)$ \\
Sum of groups & $503(100 \%)$ & $10(1.98 \%)$ \\
\hline
\end{tabular}

Table 8-Association between breastfeeding duration and prevalence of anterior open bite, with odds ratio (OR) calculation, in children without history of pacifier and/or finger-sucking habits $(n=503)$

\begin{tabular}{lcccc}
\hline Comparisons & $\chi^{2}$ & p value & OR & p value \\
\hline G1/G2 & 3.78 & 0.052 & - & - \\
G1/G3 & 2.77 & 0.096 & - & - \\
G1/G4 & 6.76 & 0.009 & 9.30 & $0.009^{* *}$ \\
G2/G3 & 0.06 & 0.806 & - & - \\
G2/G4 & 0.39 & 0.532 & - & - \\
G3/G4 & 0.73 & 0.391 & - & - \\
\hline
\end{tabular}

G1: non-breastfed. G2: shorter than 6 months. G3: interruption between 6 and 12 months. G4: longer than 12 months. c2: chi-square value. ${ }^{* *}$ : $p$ value $<0.01$ 
In the No habits group, only $10(1.98 \%)$ children had anterior open bite and the relative frequency of this malocclusion was higher in G1 (Table 7). Conversely, a relatively lower percentage of anterior open bite was observed in G4. The comparison tests indicated a significant difference between $\mathrm{G} 1$ and G4 (Table 8). Non-breastfed children would have a 9.3 times greater chance of presenting anterior open bite, compared to those who were breastfed for a period longer than 12 months.

\section{DISCUSSION}

Anterior open bite in primary dentition has been associated with nutritive (bottle-feeding) and nonnutritive sucking habits $2,5,17$. Clinical interest in the etiology and early diagnosis of this malocclusion justifies the epidemiological investigations, considering that anterior open bite may require professional assistance during the primary dentition stage by counseling on discontinuation of sucking habits, associated with interceptive orthodontic treatment or not. Frequently, some intervention is needed not only to prevent dentoskeletal alterations, but also to eliminate perpetuating factors that modify swallowing pattern and speech, such as tongue interposition between the incisors.

For the total sample, prevalence of anterior open bite was greater in non-breastfed children $(31.9 \%)$, being gradually lower in the breastfed groups. Legovic and Ostric ${ }^{11}$ (1991) recorded a frequency of $34.2 \%$ for non-breastfed children. According to Table 1, children who were breastfed for longer than 12 months had a fivefold lower frequency of anterior open bite in comparison with those who were not breastfed. Moreover, in the No habits group (Table 7), non-breastfed children exhibited a ninefold higher prevalence of anterior open bite compared with those who were breastfed for longer than 12 months (9.09\% versus $1.06 \%$ ). Considering the overall results, it may be assumed that the present study clearly demonstrated an inverse relationship between breastfeeding duration and prevalence of anterior open bite, not only in the total sample, but also in the subsample of children without history of non-nutritive sucking (Tables 2, 3 and 8 and Figure 1). The logistic regression model shown in Table 3 allows the calculation of distinct OR values by eligible breastfeeding durations, and in Figure 1, the relative probabilities may be obtained. Notably, breastfeeding for 12 months was associated with a relatively low probability (about $12 \%$ ) of occurrence of anterior open bite (Figure 1). By means of the mathematical equation derived from the logistic regression model on Table 3 [OR=exponential(coefficient $X$ time in months); $2.718(0.11 \times 12)=3.7$ ], prolonging breastfeeding for 12 months may be associated with a 3.7 times lower chance of having anterior open bite. Analysis of the association between breastfeeding and prevalence of anterior open bite indicated that breastfeeding practice interrupted between 6 and 12 months of age, or beyond 12 months of age, positively influenced dental occlusion, when compared with the complete absence of breastfeeding. However, breastfeeding for longer than 12 months of age had by far the best effect (Tables 2 and 8 ).

As would be expected, persistent non-nutritive sucking habits also had an outstanding influence on the prevalence of anterior open bite (Table 6 and Figure 2), corroborating the findings of other studies $1,2,12,15,17,21$. For each additional year of persistence with non-nutritive sucking, there was a 2.38 times greater chance of having anterior open bite. However, dental occlusion may be affected by several genetic and environmental factors. In children without history of non-nutritive sucking habits, a North American study ${ }^{1}$ and another Brazilian investigation ${ }^{25}$ recorded prevalence values for anterior open bite of $0 \%$ and $1 \%$, respectively. In South Kanara (India) ${ }^{5}$ and Iowa (USA) ${ }^{2}$, among children without history of non-nutritive sucking habits, frequencies related to anterior open bite were $5.25 \%$ and $6.1 \%$, respectively.

Comparative assessments of the influence of breastfeeding and non-nutritive sucking behaviors should take into account the time magnitude of these two variables. In general, breastfeeding does not last for a period longer than 12 months. On the other hand, non-nutritive sucking habits are more frequently ceased after 2 years of age ${ }^{2,15}$. In fact, the age of persistence with non-nutritive sucking habits does not correspond exactly to their duration. However, the pacifier is generally introduced at very early ages ${ }^{7}$, and in Brazil, pacifier-sucking has been recorded with a much higher frequency when compared with finger-sucking ${ }^{20}$. Some authors stated that, for statistical purposes, the age of persistence with the pacifier-sucking habit was more meaningful than the age of beginning to use the dummy ${ }^{17}$. Therefore, the age at which the habit ceased would be a suitable indicator in retrospective studies with the goal of assessing the associations between non-nutritive sucking habits and the development of dental occlusion.

Warren and Bishara22 (2002) had already mentioned the difficulty of analyzing the effects of breastfeeding on the development of the dental arches, because of the need for separating these effects from those of non-nutritive sucking habits. In this study, the effect of different breastfeeding patterns was also examined as an isolated factor. Non-breastfed children would be 9.3 times more prone to present anterior open bite in relation to children who received breastfeeding for periods 
longer than 12 months of age (Table 8). Presumably, most non-breastfed children in the No habits group were bottle-fed. Viggiano, et al. ${ }^{21}$ (2004) observed that $14 \%$ of exclusively bottle-fed children or those who had artificial feeding introduced in the first 3 months of life presented anterior open bite. Ovsenik $^{15}$ (2009) mentioned that bottle-feeding contributes to an atypical swallowing pattern that may have harmful influences on the developing occlusion. The use of an artificial nipple for infant feeding may be implicated in the development of anterior open bite. Perhaps, if there was a larger number of children without non-nutritive sucking habits in the sample, it would have been possible to demonstrate a grading in the intensity of the association between distinct breastfeeding duration patterns and the occurrence of anterior open bite.

From a clinical perspective, this study identified the degree of interplay among breastfeeding duration, non-nutritive sucking and prevalence of anterior open bite. Prolonged breastfeeding practice was not only significantly associated with smaller chances of acquiring non-nutritive sucking habits (Tables 4 and 5), but also with lower chances of having anterior open bite in primary dentition (Tables 7 and 8). Therefore, prolonged breastfeeding may exert a positive effect on the development of overbite in primary dentition, not only by satisfying the urge to suck and thus preventing the acquisition of non-nutritive sucking habits, but also possibly stimulating the proper tongue position and nose breathing.

Supportive advice on breastfeeding should be intensified during pregnancy and puerperium, including preventive dental visits even before the child's first tooth appears ${ }^{18}$. Prolonging breastfeeding is related to the gradual introduction of semisolid and solid foods in the child's diet, as the dental eruption process takes place, but eliminates the need to use a feeding bottle. The WHO has advised against offering artificial nipples (bottle and pacifier), because of the possibility of associations with early weaning and establishment of malocclusions ${ }^{23}$. This epidemiological investigation, by working with a sufficiently large sample, combined with enhanced selection criteria, careful division of the study groups and adequate assessment methods, demonstrated the fundamental basis of this argument for promoting breastfeeding. In a systematic review requested by $\mathrm{WHO}^{9}$, the authors stated that there are no benefits of introducing complementary foods between 4 and 6 months of age. The available evidence has demonstrated no apparent risks in recommending, as a general policy, exclusive breastfeeding for the first 6 months of life in both developing and developed country settings $\mathrm{s}^{9,24}$.

There are some limitations when evaluating the findings of this research. The studied sample must not be considered an effective representation of the whole Brazilian population, given the remarkable geographical extension and the multitude of ethnic and cultural peculiarities of this country. The retrospective design of this investigation did not avoid recall bias. In fact, as in many other studies, data collection on infant feeding and non-nutritive sucking behaviors relied on maternal recall of information. However, the young age of the children enhanced the likelihood that the information yielded while filling out the different items of the questionnaire was reliable. Furthermore, a pilot test with the questionnaire used ascertained a suitable degree of reliability.

Because the positive influence of breastfeeding may be masked by the effect of exposure to persistent non-nutritive sucking habits, it is suggested that future studies should be planned with a multicenter design, comprising similar or larger samples. A longitudinal design would be more appropriate for validating the actual effect of breastfeeding on dental occlusion. Nevertheless, the demanding and time-consuming task, as well as the broad sample required for this type of assessment, makes it somewhat difficult to follow-up many individuals for a long period.

Despite the methodological limitations, this study represents a valid contribution to promoting breastfeeding. Mothers should be encouraged to breastfeed their children for periods as long as reasonably possible, considering the biological (maternal and infant) and logistic conditions that are implicated in this protective act.

\section{CONCLUSIONS}

Persistent non-nutritive sucking habits were significantly associated with greater chances of having anterior open bite. Furthermore, inverse relationships were observed between breastfeeding duration and the prevalence of non-nutritive sucking habits, as well as breastfeeding duration and the prevalence of anterior open bite;

The chances of diagnosing anterior open bite were significantly greater for non-breastfed children in comparison with those breastfed for periods longer than 12 months, even in children without a history of non-nutritive sucking habits;

Therefore, prolonged breastfeeding practice, with total avoidance of bottles and artificial nipples, seems to exert very positive effects not only by preventing the acquisition of non-nutritive sucking habits, but also by directly stimulating proper oral development and dental occlusion. 


\section{REFERENCES}

1- Adair SM, Milano M, Lorenzo I, Russel C. Effects of current and former pacifier use on the dentition of 24- to 59-month-old children. Pediatr Dent. 1995; 17:437-44.

2- Bishara SE, Warren JJ, Broffitt B, Levy SM. Changes in the prevalence of nonnutritive sucking patterns in the first 8 years of life. Am J Orthod Dentofacial Orthop. 2006;130:31-6.

3- Degano MP, Degano RA. Breastfeeding and oral health. A primer for the dental practitioner. N Y State Dent J. 1993;59:30-2.

4- Foster TD, Hamilton MC. Occlusion in the primary dentition. Study of children at 2 and one-half to 3 years of age. Br Dent J. 1969;126:76-9.

5- Ganesh M, Tandon S, Sajida B. Prolonged feeding practice and its effects on developing dentition. J Indian Soc Pedod Prev Dent. 2005;23:141-5.

6- Gomes CF, Trezza EM, Murade EC, Padovani CR. Surface electromyography of facial muscles during natural and artificial feeding of infants. J Pediatr (Rio de Janeiro). 2006;82:103-9.

7- Howard CR, Howard FM, Lanphear B, Eberly S, deBlieck EA, Oakes $D$, et al. Randomized clinical trial of pacifier use and bottlefeeding or cupfeeding and their effect on breastfeeding. Pediatrics. 2003;111:511-8.

8- Karjalainen S, Rönning O, Lapinleimu H, Simell O. Association between early weaning, non-nutritive sucking habits and occlusal anomalies in 3-year-old Finnish children. Int J Paediatr Dent. 1999;9:169-73.

9- Kramer MS, Kakuma R. The optimal duration of exclusive breastfeeding: a systematic review. Geneva: WHO; 2002. [cited 2007 Jan 6]. Available at: http://www.who.int/nutrition/ publications/optimal_duration_of_exc_bfeeding_review_eng.pdf. 10- Labbok MH, Hendershot GE. Does breast-feeding protect against malocclusion? An analysis of the 1981 Child Health Supplement to the National Health Interview Survey. Am J Prev Med. 1987;3:227-32.

11- Legovic $M$, Ostric $L$. The effects of feeding methods on the growth of the jaws in infants. J Dent Child. 1991;58:253-5.

12- López Del Valle LM, Singh GD, Feliciano N, Machuca MC. Associations between a history of breast feeding, malocclusion and parafunctional habits in Puerto Rican children. P R Health Sci J. $2006 ; 25: 31-4$
13- Meyers A, Hertzberg J. Bottle-feeding and malocclusion: is there an association? Am J Orthod Dentofacial Orthop. 1988; 93:149-52.

14- Moimaz SA, Zina LG, Saliba NA, Saliba O. Association between breast-feeding practices and sucking habits: a cross-sectional study of children in their first year of life. J Indian Soc Pedod Prev Dent. 2008;26:102-6.

15- Ovsenik $M$. Incorrect orofacial functions until 5 years of age and their association with posterior crossbite. Am J Orthod Dentofacial Orthop. 2009;136:375-81.

16- Peres KG, Barros AJ, Peres MA, Victora CG. Effects of breastfeeding and sucking habits on malocclusion in a birth cohort study. Rev Saude Publica. 2007;41:343-50.

17- Peres KG, Oliveira Latorre MR, Sheiham A, Peres MA, Victora CG, Barros FC. Social and biological early life influences on the prevalence of open bite in Brazilian 6-year-olds. Int J Paediatr Dent. 2007; 17:41-9.

18- Rezende GPSR, Costa LRRS, Cardoso RA. Pediatric dentistry during rooming-in care: evaluation of an innovative project for promoting oral health. J Appl Oral Sci. 2004;12:149-53.

19- Righard L. Early enhancement of successful breast-feeding. World Health Forum. 1996;17:92-7.

20- Scavone-Jr H, Guimarães CH Jr, Ferreira RI, Nahás ACR, VelliniFerreira F. Association between breastfeeding duration and nonnutritive sucking habits. Community Dent Health. 2008;25:161-5. 21- Viggiano D, Fasano D, Monaco G, Strohmenger L. Breast feeding, bottle feeding, and non-nutritive sucking; effects on occlusion in deciduous dentition. Arch Dis Child. 2004;89:1121-3. 22- Warren JJ, Bishara SE. Duration of nutritive and nonnutritive sucking behaviors and their effects on the dental arches in the primary dentition. Am J Orthod Dentofacial Orthop. 2002;121:34756.

23- World Health Organization. Division of Child Health and Development. Evidence for the ten steps to successful breastfeeding. Geneva: WHO; 1998.

24- WHO Multicentre Growth Reference Study Group. Breastfeeding in the WHO Multicentre Growth Reference Study. Acta Paediatr Suppl. 2006;450:16-26.

25- Zardetto CG, Rodrigues CR, Stefani FM. Effects of different pacifiers on the primary dentition and oral myofunctional structures of preschool children. Pediatr Dent. 2002;24:552-60. 